\title{
Dynamics of the plumes produced by ultrafast laser ablation of metals
}

\author{
T. Donnelly, ${ }^{1}$ J. G. Lunney, ${ }^{1}$ S. Amoruso, ${ }^{2, a)}$ R. Bruzzese,${ }^{2}$ X. Wang ${ }^{2}$ and X. $\mathrm{Ni}^{3}$ \\ ${ }^{1}$ School of Physics, Trinity College Dublin, Dublin 2, Ireland \\ ${ }^{2}$ Dipartimento di Scienze Fisiche and CNR-SPIN, Università degli Studi di Napoli Federico II, Complesso \\ Universitario di Monte S. Angelo, Via Cintia, I-80126 Napoli, Italy \\ ${ }^{3}$ Department of Electronic Engineering, Tianjin University of Technology and Education, Tianjin 300222, \\ People's Republic of China
}

(Received 4 March 2010; accepted 3 July 2010; published online 30 August 2010)

\begin{abstract}
We have analyzed ultrafast laser ablation of a metallic target (Nickel) in high vacuum addressing both expansion dynamics of the various plume components (ionic and nanoparticle) and basic properties of the ultrafast laser ablation process. While the ion temporal profile and ion angular distribution were analyzed by means of Langmuir ion probe technique, the angular distribution of the nanoparticulate component was characterized by measuring the thickness map of deposition on a transparent substrate. The amount of ablated material per pulse was found by applying scanning white light interferometry to craters produced on a stationary target. We have also compared the angular distribution of both the ionic and nanoparticle components with the Anisimov model. While the agreement for the ion angular distribution is very good at any laser fluence (from ablation threshold up to $\approx 1 \mathrm{~J} / \mathrm{cm}^{2}$ ), some discrepancies of nanoparticle plume angular distribution at fluencies above $\approx 0.4 \mathrm{~J} / \mathrm{cm}^{2}$ are interpreted in terms of the influence of the pressure exerted by the nascent atomic plasma plume on the initial hydrodynamic evolution of the nanoparticle component. Finally, analyses of the fluence threshold and maximum ablation depth were also carried out, and compared to predictions of theoretical models. Our results indicate that the absorbed energy is spread over a length comparable with the electron diffusion depth $L_{c}(\approx 30 \mathrm{~nm})$ of $\mathrm{Ni}$ on the timescale of electron-phonon equilibration and that a logarithmic dependence is well-suited for the description of the variation in the ablation depth on laser fluence in the investigated range.
\end{abstract}

(C) 2010 American Institute of Physics. [doi:10.1063/1.3475149]

\section{INTRODUCTION}

Ultrashort laser ablation (ULA) of metals using femtosecond (fs) pulses at intensities $\sim 10^{12}-10^{13} \mathrm{~W} / \mathrm{cm}^{2}$ is currently receiving much attention both in terms of understanding the ablation physics and informing the development of new material processing techniques. ULA differs in several ways from nanosecond (ns) laser ablation. Since the laser pulse duration is much shorter than the electron-phonon relaxation time, $\tau_{e p}$ (typically $\approx 10 \mathrm{ps}$ for elemental metals), there is little hydrodynamic movement of the heated material during the laser pulse. During the laser pulse the electrons within the optical penetration depth are driven to high temperature while the lattice remains relatively cold. On time scale of $\tau_{e p}$ the electron temperature drops through energy relaxation to the lattice. Energy continues to be transported deeper in the target by electron heat conduction and ablation of part of the heated region ensues.

Much of the research on ULA has concentrated on the laser absorption, electron-phonon relaxation, electron heat conduction and phase decomposition leading to ablation. The two-temperature model (TTM) provides a good description of the thermal history of the electron and lattice subsystems. ${ }^{1}$ Hydrodynamic model (HM) and molecular dynamic (MD) simulations have been both used to describe the thermodynamic evolution leading to ablation. ${ }^{2-9}$ These simulations

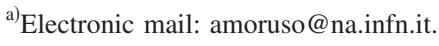

show that the overall ULA process is governed by a set of rather complex mechanisms occurring on very fast time scales and varying on small spatial scales. The initial laser heating is nearly isochoric and the relaxation of the hot nearsolid-density material proceeds in a different manner at different depths in the target. Near the surface the material is completely atomised; deeper layers decompose into a mixture of vapor and liquid clusters through mechanical fragmentation and phase explosion. This leads to the appearance of two distinct ablation plumes in the far-field: a fast-moving atomic plume which can be partially ionised and a slowermoving nanoparticle (NP) plume, as observed in several experiments. ${ }^{10-12}$ Typically, NPs constitute the major part of the ablated material, and the atomic plume accounts for only $10 \%-20 \%$ of the total ablated mass. ${ }^{3,5,12,13}$ Recently, ultrafast time-resolved x-ray absorption spectroscopy (with $30 \mathrm{ps}$ time resolution) during ULA of a metallic target (Al), in vacuum, showed that a structured nascent plume, with the faster atomic species followed by a hot-liquid-phase of slower NPs, already exists at the very early stages ( $\leq 30 \mathrm{~ns})$ of the ablation process. ${ }^{14}$ This study also indicated that material ejection from the target surface starts within the first $30 \mathrm{ps}$ and lasts for few tens of ns.

The properties of the ablated material and its expansion dynamics are of interest in numerous research fields, such as pulsed laser deposition (PLD), laser induced breakdown spectroscopy, ion beam generation, NP synthesis and deposition. Analysis of the expansion dynamics can also improve 
the physical understanding of the overall ablation process. It is, therefore, interesting to relate parameters of the ejected plumes in the far-field, for times of few microseconds or more and distances from millimeters to centimeters from the target surface, to those near the target at the early stages of ablation. The large differences in the spatial and temporal scales involved make the use of HM codes and MD simulations unfeasible, thus, suggesting the need to consider models which are suitable for the different stages and try to match them at an intermediate phase. Besides, in the very early stage a one-dimensional description is typically employed, while it should be considered that some time after ablation the ejection of material ceases and the expansion of the clouds of ablated particles becomes fully threedimensional (3D). At this late stage more conventional hydrodynamic descriptions can be used to describe the expansion of the ejected species. One of these is the adiabatic isentropic expansion model of Anisimov et al. ${ }^{15,16}$ which has been extensively applied to PLD with ns pulse duration to describe both the laser ablation plume propagation and the angular profile of the deposition rate. ${ }^{17-19}$ These studies indicate that the Anisimov model provides a rather good description of how the ablated material expands from a small, hot, dense vapor cloud at the end of the ns laser pulse to the time when the ablated material has traveled several centimeters, or more, from the target. More recently, the Anisimov model was also shown to describe fairly well the angular distribution of the ionised plume produced by ULA of metallic thin films or bulk targets. ${ }^{19,20}$ In contrast, the application of the Anisimov model to describe the expansion of the NP plume in ULA has not yet been explored.

In this paper we describe the results of some experiments to examine various aspects of the ablation plume expansion in ULA of metals. We have mainly sought to explore the extent to which the Anisimov model can describe the expansion of both the atomic and NP plumes produced in the ULA process. This will help to highlight any differences between the ablation components and provide insights into the geometrical characteristics of these plumes at the beginning of their 3D expansion, which are of paramount interest in view of the matching of the late expansion stage with the predictions of complex models of the early ablation phase. Finally, we compare the measured ablation rate with earlier models of laser ablation based on the TTM, ${ }^{21,22}$ trying to address some aspects which have not yet been considered so far.

The paper is divided in several sections. The following section describes the experimental methods, while Sec. III reports the theoretical background of the Anisimov model, and some predictions of the plume expansion characteristics in our experimental conditions. Sec. IV is devoted to the experimental characterization of the ion and NP plumes expansions, and to their discussion within the framework of the Anisimov model. Sec. V presents an analysis of the ablation rate as a function of the laser fluence and a comparison with predictions of simple models generally employed to describe the ablation threshold and dependence of the ablation rate on

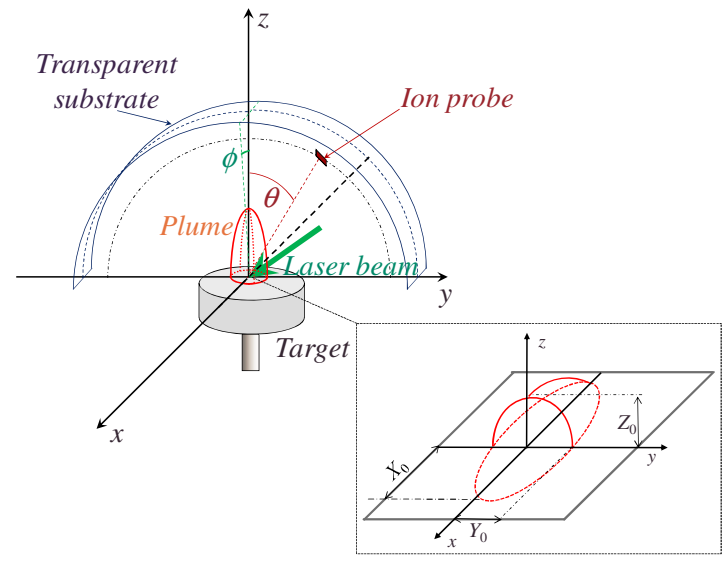

FIG. 1. (Color online) Schematic of the experimental setup. The ion probe moves on a circle in the $z y$ plane centered at the ablation spot. The ablated material is collected on a circularly-curved transparent plastic substrate also centered at the ablation spot. Sections of the deposits in the $z y$ and $z x$ planes are used to obtain angular deposition profiles as a function of the angle $\theta$ on a hemispherical surface and of the angle $\phi$ on a planar surface. The shape of the ablation plume is shown. The inset shows the semiellipsoidal initial plume with the indication of the initial dimensions $X_{0}, Y_{0}$, and $Z_{0}$ along the three axes.

material physical parameters. Finally, Sec. VI summarizes the present investigations and outlines the main conclusions which can be drawn.

\section{EXPERIMENTAL METHODS}

The experimental setup used in this study is schematically depicted in Fig. 1. The diagnostic setup is placed in a high vacuum chamber and comprises an ion probe, which can be moved on a circle in the zy plane, and a circularlycurved transparent plastic substrate collecting the ablated material. Both the ion probe and the substrate are centered at the ablation spot. In Fig. 1 the shape of a semiellipsoidal plume expanding toward the substrate is also drawn schematically. The inset shows a magnification of the semiellipsoidal initial plume with the indication of the initial dimensions $X_{0}, Y_{0}$, and $Z_{0}$ along the three axes, which will be used in the Anisimov model of Sec. III.

The laser source delivers $\approx 250 \mathrm{fs}, 1 \mathrm{~mJ}$ laser pulses at $527 \mathrm{~nm}$, at a repetition rate of $33 \mathrm{~Hz}$. The laser beam intensity profile was approximately Gaussian. The $p$-polarized laser beam was focused at $45^{\circ}$ incident angle to an elliptical spot on a Ni target in high vacuum at $10^{-7}$ mbar. The laser beam intensity profile on the target was found by measuring the major and minor diameters of elliptical burn spots as a function of laser pulse energy, $E$, and evaluating the slope on a semilogarithmic plot. ${ }^{23}$ Two laser shots were fired at each location and the damaged area was measured by an optical microscope. The estimated spot size was $\sigma_{0}=1.3$ $\times 10^{-3} \mathrm{~cm}^{2}$. The peak fluence at the center of the beam is given by $F_{p}=2 E / \sigma_{0}$, which, for a maximum energy on target $E \approx 500 \mu \mathrm{J}$, gives $F_{p} \approx 0.8 \mathrm{~J} / \mathrm{cm}^{2}$. The average fluence within a spot of area $\sigma_{0}$ is $F=E / \sigma_{0}=0.5 F_{p}$.

The plasma part of the ablation plume was measured using a planar Langmuir probe, ${ }^{24}$ oriented to face the target spot and located at a distance of $64 \mathrm{~mm}$ from the target (see Fig. 1). The probe collecting area was a $2.5 \times 5 \mathrm{~mm}^{2}$ copper 
plate insulated at the rear side. The probe was negativelybiased to reject electrons, thus, the current density recorded by the probe is directly related to the positive ion flux due to the plasma flow at the probe position. The angular distribution of the ion flux in the atomic plume was measured by moving the probe on a circle in the $z y$ plane (see Fig. 1). A second fixed probe $\left(2 \times 2 \mathrm{~mm}^{2}\right)$ at the same distance and close to the target normal was used to ensure the ablation conditions remained constant. 64 ion signals were averaged for each condition.

To examine the angular distribution of ablated material, which is predominately NPs, ${ }^{3,5,12,13}$ we measured the spatial distribution of deposition on the circularly-curved transparent plastic substrate which was positioned at $77 \mathrm{~mm}$ from the ablation spot and centered on it. The film thickness was found by measuring the optical transmission, around $515 \mathrm{~nm}$, of the Ni film using a calibrated flatbed scanner. UV-visible spectrophotometry and spectroscopic ellipsometry of the $\mathrm{Ni}$ films showed that the optical constants are close to the bulk values, therefore, the film thickness was derived from the measured transmission using the optical constants of bulk Ni. This deposition technique allows the angular distribution of ablated material to be obtained in the $z y$ plane, as a function of the angle $\theta$ on a hemispherical surface, and in the $z x$ plane, as a function of the angle $\phi$ on a planar surface (see Fig. 1). These can be directly compared to the Anisimov model predictions. ${ }^{15-17}$ During ion probe and deposition measurements the Ni target was rotated in its own plane to avoid drilling a hole.

Finally, craters were produced delivering a given number of laser pulses at the same position on a stationary target. The amount of ablated material per pulse as a function of the laser fluence was obtained by measuring the craters with a Zygo scanning white light interferometer.

\section{THEORETICAL BACKGROUND}

The Anisimov model provides a gas dynamical description of the adiabatic phase of expansion of a laser ablation plume in vacuum. In ns laser ablation this adiabatic regime commences at the end of the laser pulse, at which time the ablated material exists as a thin layer of hot vapor on the surface of the target (see inset of Fig. 1). It is based on a 3D, adiabatic and isentropic self-similar solution of the gas dynamics equations. While the model was developed for the expansion of a neutral gas cloud, it has been successfully used to describe the expansion of both the plasma part and the overall plume (ions and neutrals) for laser produced plasmas (LPP) generated by ns laser ablation in vacuum. ${ }^{17,18}$ The expansion is considered to be isentropic when the thermal diffusion is too slow to keep pace with the rate of expansion. Singh and Narayan ${ }^{25}$ considered the case where the plume can be considered to be isothermal. By comparing the expansion velocity and the rate of heat diffusion in a LPP, Lunney et $a .^{26}{ }^{26}$ estimated that when the electron temperature is less than $\sim 12 \mathrm{eV}$ the expansion is isentropic.

The thickness of the layer of ablated material at the onset of the adiabatic expansion is a critical parameter in the Anisimov expansion model, since it determines the extent to which the final plume is forward-peaked. For ns pulses, vapor evolved from the target during the laser pulse is further heated by absorption of the laser. Thus, apart from some energy transport from the vapor to the target, the end of the laser pulse seems the appropriate choice for the onset of the adiabatic expansion. The initial dimensions of the gas cloud can then be estimated as follows. The initial thickness $d$ is approximated as $d \approx c_{s} \tau_{p}$ where $c_{s}$ is the sound velocity and $\tau_{p}$ is the laser pulse duration. The dimensions in the plane of the target are determined by the size of the ablation spot, which is related to the laser beam spot. Good agreement with the experimental data seems to support these assumptions. $^{17,18}$

For ULA it is not clear how to choose the initial thickness of the ablated material at the beginning of the adiabatic expansion. It was suggested earlier that the initial longitudinal dimension of an ULA plume could be taken as comparable to the thickness of removed material. ${ }^{2}$ This would result in an extremely narrow angular width of the plume in the inertial expansion stage, which was not observed experimentally. ${ }^{19,20}$ Indeed, it has been observed that two distinct plumes are produced in the ULA process, a fastermoving atomic plume and a slower NP one.$^{10-12}$ The validity of the Anisimov model should be tested for both components, which is the main aim of the present work.

Looking first at the formation of the atomic plume, we can note that at the end of the fs laser pulse the material near the target surface has a high electron temperature, but the density is still close to the solid value. A rarefaction wave will move into the hot material at about the sound velocity and vapor will move away from the target with a maximum velocity of $2 c_{s} /(\gamma-1)$, where the adiabatic index $\gamma$ has a value of about 1.25 in a low temperature plasma. ${ }^{27}$ The formation of the vapor layer will cease when the rarefaction wave reaches a depth beyond which the material is not transformed into vapor. At the fluence level of concern to the present experiment, this depth corresponds to $10 \%-20 \%$ of the total ablation depth, ${ }^{3,5,12,13}$ and amounts to $10-20 \mathrm{~nm}$ at the highest values of fluence used here. Considering the part of laser pulse energy absorbed in such superficial layer, at $F_{p} \approx 0.6 \mathrm{~J} / \mathrm{cm}^{2}$ an absorbed energy per atom of $\approx 10-15 \mathrm{eV}$ can be estimated, which gives $c_{s} \approx(2-3)$ $\times 10^{3} \mathrm{~m} / \mathrm{s}$. Thus a formation time for the atomic vapor layer of $\approx 3-7$ ps is obtained. This, in turn, leads to a lower limit of $\approx 50-200 \mathrm{~nm}$ for the initial thickness of the atomic vapor when the adiabatic expansion begins. This estimate is consistent with recent work on double pulse ULA of Ni which showed that when the second pulse is delayed beyond $\approx 10 \mathrm{ps}$ it is primarily absorbed in the vapor produced by the first pulse and does not contribute to further ablation. ${ }^{20}$

Regarding the definition of the parameters which determine the expansion of the NP plume, the situation is even less clear than for the atomic plume. However, time-resolved images of NP plume expansion show that they are quite ellipsoidal in shape ${ }^{11}$ as is the case in the Anisimov model. The starting thickness of the adiabatic expansion of the NP layer might not be smaller than the ablation depth, but the appropriate value of $\gamma$ for a NP plume is not clear at this stage. 
The Anisimov model has been described in detail elsewhere, ${ }^{15-18}$ thus, only a brief description will be given here and related to our experimental conditions. The initial plume is considered to be semiellipsoidal in shape with principal radii $X_{0}, Y_{0}$, and $Z_{0}$, where $X_{0}$ and $Y_{0}$ are the major and minor radii of the ablation spot and $Z_{0}$ is the thickness of the ablated material, as shown in the inset of Fig. 1. The $x$ axis is chosen to lie along the major radius of the beam spot. As the expansion proceeds the radii of the ellipsoidal plume front in the $x, y$ and $z$ directions are $X(t), Y(t)$, and $Z(t)$. The plume propagation is described by the following set of equations:

$$
\xi \frac{d^{2} \xi}{d \tau^{2}}=\eta \frac{d^{2} \eta}{d \tau^{2}}=\zeta \frac{d^{2} \zeta}{d \tau^{2}}=\left(\frac{\xi_{0} \eta_{0} \zeta_{0}}{\xi \eta \zeta}\right)^{\gamma-1},
$$

where

$$
\xi=\frac{X(t)}{X_{0}} ; \quad \eta=\frac{Y(t)}{X_{0}} ; \quad \zeta=\frac{Z(t)}{X_{0}} ; \quad \tau=\frac{t}{t_{0}}
$$

are dimensionless space and time variables. The time parameter $t_{0}$ is given by

$$
t_{0}=\frac{\sqrt{\beta}}{X_{0}} \text {. }
$$

The parameter $\beta$ is defined as

$$
\beta=(5 \gamma-3) \frac{E_{p}}{M_{p}}=(5 \gamma-3) \frac{\varepsilon_{p}}{m_{p}},
$$

where $E_{p}$ and $M_{p}$ are the thermal energy and mass of the initial plume and $\varepsilon_{p}$ and $m_{p}$ are the average energy per particle and mass of the plume particles. It is worth nothing that while $t_{0}$ and, therefore, $\beta$ define the time scale of the plume expansion, the plume shape in the inertial expansion stage $\left(t \gg t_{0}\right)$ does not depend on these parameters. Numerical solution of this equation set yields the temporal variation in the principal radii of the plume front and, therefore, the plume shape.

The plume expansion behavior can be illustrated by choosing starting parameters of the same order as for the plasma plume in our ULA experiment, namely $Y_{0} / X_{0}=0.5$. In our experiment $X_{0}$ and $Y_{0}$ are approximately $200 \mu \mathrm{m}$ and $100 \mu \mathrm{m}$, respectively (see Sec. III). The initial plume thickness, $Z_{0}$, is set to $100 \mathrm{~nm}, \varepsilon_{p}=15 \mathrm{eV}, m_{p}=58.70 \mathrm{u}$ is the atomic mass of nickel (Ni), and 1.25 is used for $\gamma$. Figure 2(a) shows the evolution of the dimensionless radii. As expected the expansion velocity is greatest in the $z$ direction which is the direction of highest pressure gradient in the initial plume. It can also be seen that the plume expansion becomes inertial (constant velocity) for values of $\tau$ beyond 5 , which means a time delay after the laser pulse of about 100 ns for the values considered above. The plume velocity in each dimension can be found by differentiation. Figure 2(b) shows the aspects ratios $\zeta / \xi, \quad \zeta / \eta$, and $\eta / \xi$ $(\equiv Z / X, Z / Y, Y / X)$ as a function of $\tau$. The so-called "flipover" effect can be seen, where the aspect ratio $Y / X$ is initially less than 1 but changes to a value greater than 1 at late time in the inertial phase. As before, this behavior is a reflection of the ratio of pressure gradients in the $x$ and $y$ directions in the initial plume. The final values of $Z / X, Z / Y$, and

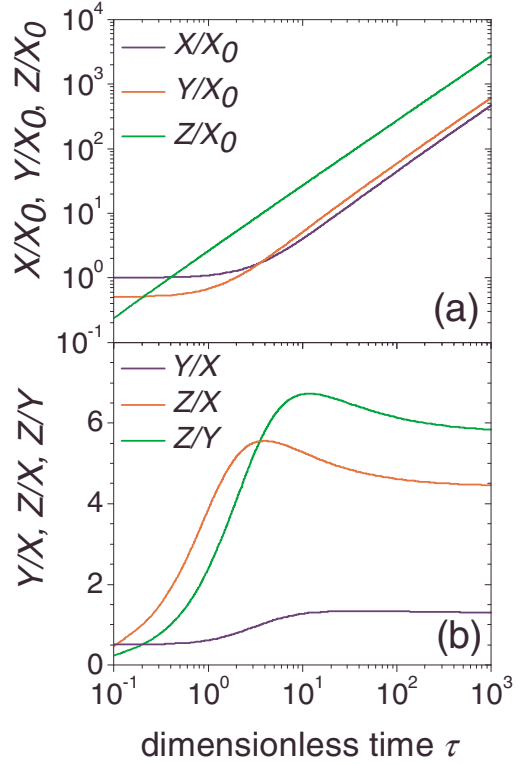

FIG. 2. (Color online) (a) Dimensionless coordinates of the plume position as a function of dimensionless time $\tau$. (b) The temporal evolution of the plume aspect ratios.

$Y / X$ are labeled $k_{z x}, k_{z y}$, and $k_{y x}$ and correspond to the plume length-to-width ratios in the $z y$ and $z x$ planes and the width ratio in the $y x$ plane. The values of $k_{z x}$ and $k_{z y}$ determine the angular spread of the particle flux on a detector at large distance and larger values correspond to more forward-peaked expansion. Figure 3 shows values of the plume aspect ratios $k_{z x}$ and $k_{z y}$ calculated as a function of normalized plume thickness $Z_{0} / X_{0}$, for $Y_{0} / X_{0}=0.5$ and for different $\gamma$ values; the upper scale shows the corresponding values of $Z_{0}$ for $X_{0}=200 \mu \mathrm{m}$. It can be seen that if the initial thickness is increased with respect to the in plane dimension, the plume aspect ratio is reduced. The plume shape is seen to depend strongly on $\gamma$, becoming more forward-peaked for larger $\gamma$ at a fixed value of $Z_{0} / X_{0}$.

The equation describing the particle flux on to a detector

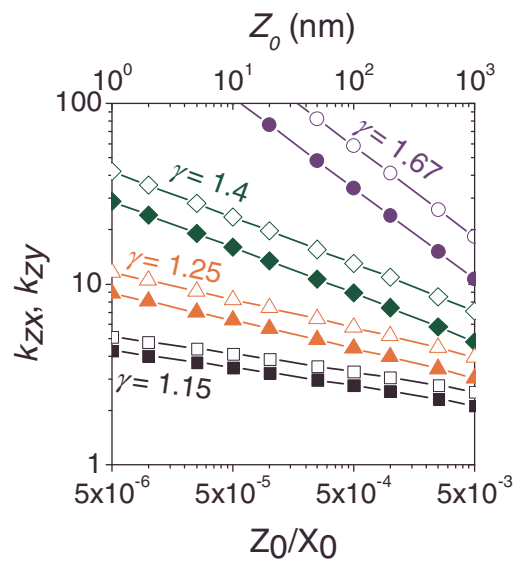

FIG. 3. (Color online) Calculation of the plume aspect ratios $k_{z x}$ (open symbol) and $k_{z y}$ (full symbol) as a function of the normalized initial plume thickness $Z_{0} / X_{0}$, for several values of the adiabatic index $\gamma$ : circles- $\gamma$ $=1.67$; diamond $-\gamma=1.4$; triangles $-\gamma=1.25$; and squares $-\gamma=1.15$. The aspect ratios were calculated for $X_{0}=200 \mu \mathrm{m}$ and $Y_{0} / X_{0}=0.5$. The upper scale reports values of $Z_{0}$. 
positioned at $(y, z)$ in the $z y$ plane and oriented to face the ablation spot is (a similar equation holds for the $z x$ plane)

$$
\begin{aligned}
j(y, z, t)= & \left(N / I_{1}\right)(X Y Z)^{-1}\left[1-\left(\frac{y}{Y}\right)^{2}-\left(\frac{z}{Z}\right)^{2}\right]^{1 / \gamma-1}\left(v_{y}^{2}\right. \\
& \left.+v_{z}^{2}\right)^{1 / 2},
\end{aligned}
$$

where $N$ is the total number of particles in the plume, $I_{1}$ is a constant which depends on the value of $\gamma$, and $v_{y}$ and $v_{z}$ are the components of the local plume velocity. ${ }^{15}$ Equation (5) describes the signal recorded on the ion probe operating in time-of-flight (TOF) mode. It also describes how the TOF signal depends on the angle $\theta$ measured away from the target normal, where $y / z=\tan \theta$. This equation can be integrated to yield the angular distribution and its associated full-widthhalf-maximum (FWHM) of the deposition on hemispherical or plane surfaces. For particles collected on a hemispherical surface, as for the ion probe or the deposit in the $z y$ plane (see Fig. 1), the angular distribution is

$$
F(\theta)=F(0)\left(1+\tan ^{2} \theta\right)^{3 / 2}\left(1+k_{z y}^{2} \tan ^{2} \theta\right)^{-3 / 2},
$$

while for particle collection on a planar surface, as in the case of the deposit profile in the $z x$ plane (see Fig. 1), it is

$$
F(\phi)=F(0)\left(1+k_{z x}^{2} \tan ^{2} \phi\right)^{-3 / 2} .
$$

The width at FWHM of the angular distribution for deposition on hemispherical and planar substrates can be found from Eqs. (6) and (7), respectively, and are as follows (see Fig. 1): ${ }^{18}$

$$
\Delta \theta=2 \arctan \sqrt{\frac{2^{2 / 3}-1}{k_{z y}^{2}-2^{2 / 3}}} ; \quad \Delta \phi=2 \arctan \sqrt{\frac{2^{2 / 3}-1}{k_{z x}^{2}}}
$$

\section{EXPANSION OF PLASMA AND NP PLUMES AND COMPARISON TO ANISIMOV MODEL}

In this section the main experimental features of the ion and NP plumes expansions will be discussed and compared to the predictions of the Anisimov model. Before discussing the experimental data on the ion TOF profiles and the angular distribution of ions and NPs, we consider useful to examine how the collected material is partitioned between ionised and nonionised material and compare it with the total number of atoms removed from the target per laser shot. This last quantity was determined by integrating crater depth maps to find the volume removed, and then dividing by the number of laser shots. As an example, Fig. 4 shows a white light interferometry map of the ablation crater produced by irradiating a stationary target with $260 \mu \mathrm{J}$ pulses for $7 \mathrm{~s}$ at $33 \mathrm{~Hz}$ (231 shots). The peak fluence in the center of the ablation spot was $F_{p} \approx 0.4 \mathrm{~J} \mathrm{~cm}^{-2}$. The maximum ablation depth per pulse is $\approx 75 \mathrm{~nm}$. Figure 5(a) shows the variation in the total amount of ablated material as a function of the peak fluence $F_{p}$. This is indicated in terms of the total number of $\mathrm{Ni}$ atoms removed from the target per laser shot, $N_{r}$, as derived from the crater depth map measurements. In Fig. 5(a) we also report the total number of atoms deposited per laser shot, $N_{d}$, as calculated from the spatial distribution of the deposits on

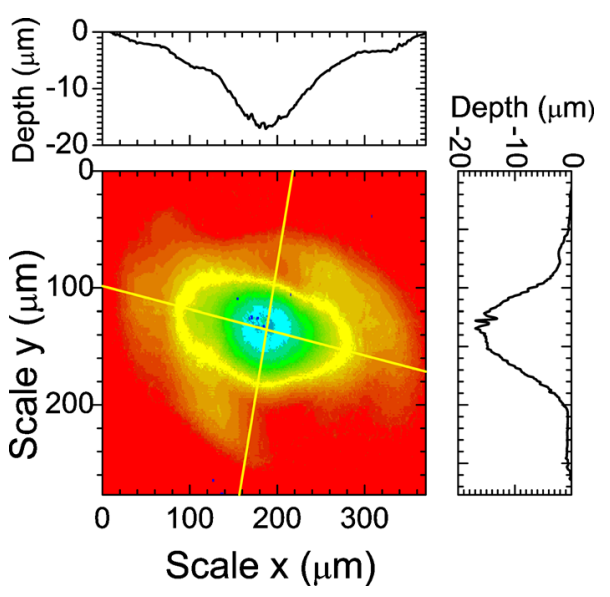

FIG. 4. (Color online) Surface profile of a crater formed from ablation at $F_{p} \approx 0.4 \mathrm{~J} \mathrm{~cm}^{-2}$. The number of shots used in this case was 231 .

the hemispherical substrate. We observe a rather good agreement between $N_{r}$ and $N_{d}$ which indicates that all the ablated material is finally deposited.

Figure 5(b) reports an approximate estimate of the total collected ion yield $N_{i}$ as a function of $F_{p}$, as obtained from the ion probe measurements. Since the ion flux angular distribution in the $z x$ plane was not measured directly, this quantity has been derived by considering an ion plume cylindrically symmetric around the target normal. The ion yield corresponds to a rather low fraction $(\leq 1 \%)$ of the total ablated material, irrespective of fluence. This is consistent with the earlier conclusion that the ablated material is predominately decomposed as NPs, while the atomic products (neutral atoms and ions) constitute a minor fraction $(\approx 10 \%-20 \%)$ of the total ablated material. ${ }^{3,5,12,13} \mathrm{We}$ can, therefore, assume in the following that the angular distribution of the deposits can be considered as representative of the NP plume. Moreover, the ion plasma plume characteristics will be illustrative of the properties of the atomic ablation component.

To compare the experimental results with the predictions

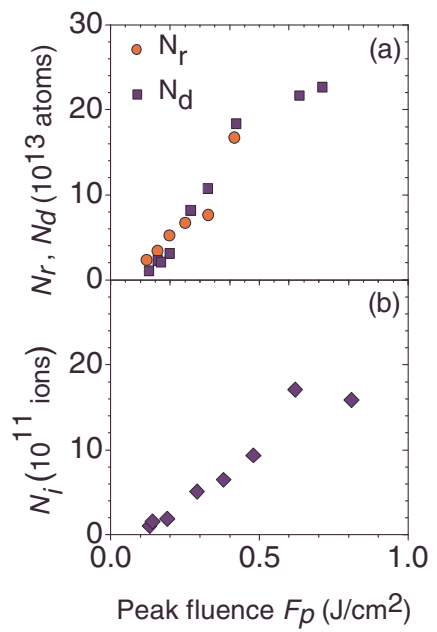

FIG. 5. (Color online) (a) Total number of atoms removed $N_{r}$ (circle) and deposited $N_{d}$ (square), per laser shot, as a function of peak fluence $F_{p}$. (b) Total number of ablated ions in the plume that was inferred from the ion probe measurements. 


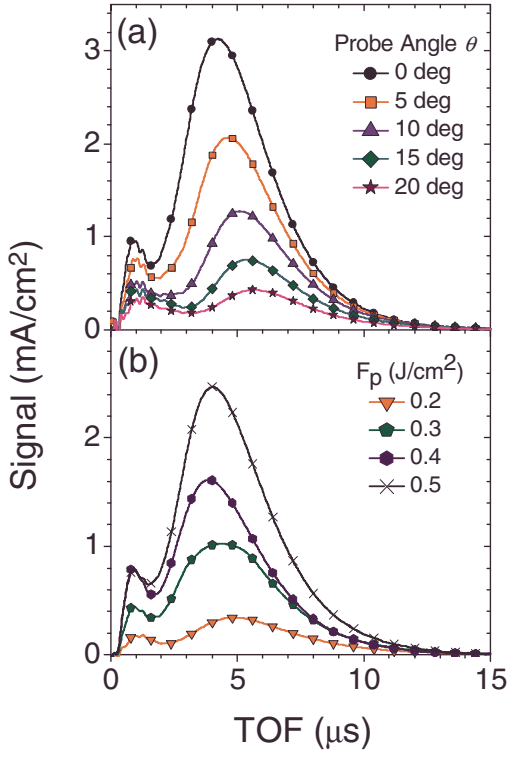

FIG. 6. (Color online) (a) Ion TOF signals for various probe angles $\theta$ at $F_{p} \approx 0.6 \mathrm{~J} \mathrm{~cm}^{-2}$. (b) Ion TOF signals for various values of the laser peak fluence $F_{p}$, measured normal to the target surface $(\theta=0)$.

of the Anisimov model, we need as input parameter for the model the transverse dimensions of the initial plume $X_{0}$ and $Y_{0}$ when the $3 \mathrm{D}$ expansion starts. In our analysis we consider for these quantities values comparable to the size of the ablated region as determined from white light interferometry map of the ablation craters, as, for example, the one shown in Fig. 4. From the analysis of crater maps obtained at different laser fluencies, we derived values of $X_{0} \approx 200 \mu \mathrm{m}$ and $Y_{0} \approx 100 \mu \mathrm{m}$, with a $15 \%$ accuracy. While an aspect ratio of 0.5 similar to that of the laser spot size $\sigma_{0}$ is retained, we observe that the area of the ablated region is almost half the damaged area, indicating that in our experimental conditions the threshold for an effective ablation is reached only on half of the irradiated region. In the following, these values of $X_{0}$ and $Y_{0}$, which were also used earlier in Sec. III, will be used to compare the experimental results to the Anisimov model predictions.

\section{A. Ion plasma plume}

The ion plasma part of the ablation plume was measured by recording the ion signal using the negatively-biased Langmuir probe. Figure 6(a) shows ion TOF signals recorded as a function of angle $\theta$ away from the target normal (see Fig. 1), for a peak fluence $F_{p} \approx 0.6 \mathrm{~J} / \mathrm{cm}^{2}$. As expected for an Anisimov-type expansion, the amplitude of the ion signal and the expansion velocity progressively fall as the detection angle $\theta$ is increased. Figure 6(b) shows ion TOF signals, measured normal to the target surface $(\theta=0)$, for various values of the peak fluence $F_{p}$. As $F_{p}$ increases, the ion signal increases accordingly, and, in general, the TOF of the peak signal is gradually reduced indicating a more energetic expansion.

There seem to be two components in the ion signal: a small amplitude fast signal followed by a much larger slow signal. The slower, second component is due to $\mathrm{Ni}$ ions formed when target regions near the surface are ablated as
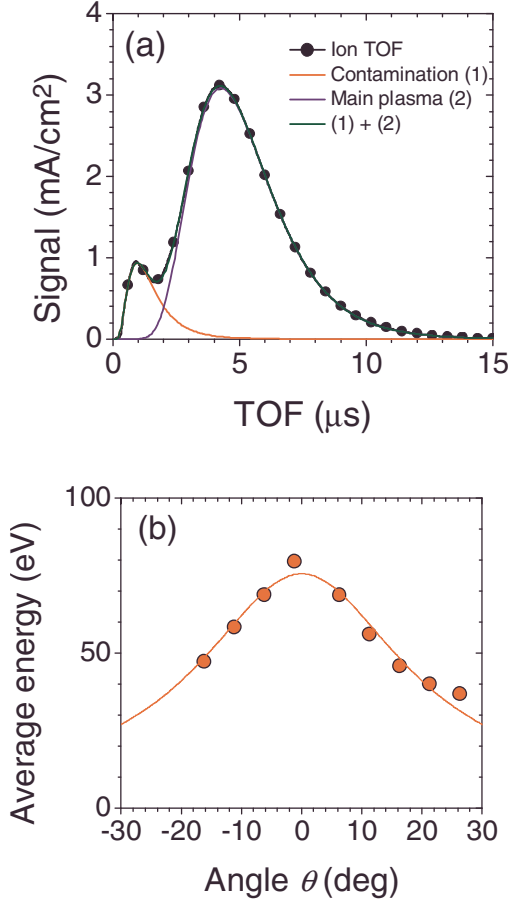

FIG. 7. (Color online) (a) Ion TOF signal normal to target at a peak fluence $F_{p} \approx 0.6 \mathrm{~J} \mathrm{~cm}^{-2}$. The signal is resolved into two separate components: the fast contamination peak and the main Ni plasma. (b) Average ion energy as a function of the probe angle $\theta$ calculated by considering the main $\mathrm{Ni}$ component of the ion TOF signals. The line is a guide for the eye.

plasma. The presence of a faster peak in ion probe signals has been previously observed in various studies of ULA of metallic targets, and assigned to low- $Z$ contamination of the target surface. ${ }^{19,28,29}$ Optical emission spectroscopy analysis of ULA of a $\mathrm{Cu}$ target has shown the presence of hydrogen emission line due to impurities, which can be reduced by appropriate target conditioning in multishot ablation experiments. ${ }^{30}$ To see if this is the origin of the fast signal in our experiment the following tests were carried out. With the laser running at $33 \mathrm{~Hz}$ and the target rotating at about $3 \mathrm{~Hz}$ the signals are as in Fig. 6. However, when the target rotation is turned off the fast signal disappears. It seems that ablation on the same spot at $33 \mathrm{~Hz}$ is sufficient to keep the surface contamination below our detection limit. Again with the target stationary, if the laser is blocked for several minutes and then unblocked, the first shot produces a large amplitude fast peak. The amplitude falls below detection over the next few shots. This behavior suggests that the contamination may derive from the vacuum chamber rather than the target itself. It can also be noted in Fig. 6 that the fast signal falls less rapidly with angle than the main peak, indicating that it has a wider angular distribution.

To simplify the analysis of the TOF signals the contamination peak was removed by fitting the overall signal with two pulse functions, as shown in Fig. 7(a), and then subtracting the fast signal. The ion energy distribution can be calculated from the ion TOF signal, and hence, the average ion energy. ${ }^{31}$ The angular variation in average ion energy is shown in Fig. 7(b) for laser peak fluence $F_{p} \approx 0.6 \mathrm{~J} / \mathrm{cm}^{2}$. The average ion energy normal to the target is greatest indicating a forward-peaked expansion in agreement with the 


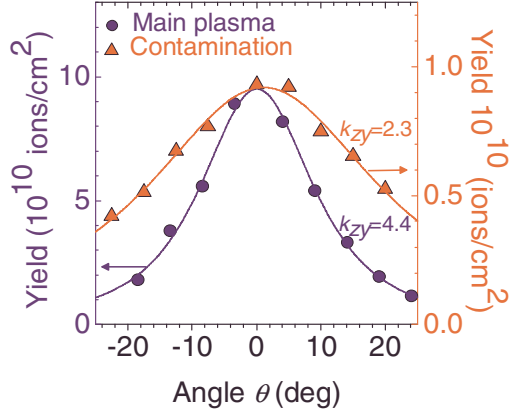

FIG. 8. (Color online) (a) Angular variation in the ion yield for the main $\mathrm{Ni}$ plasma component (circles, left axis) and for the contamination component (triangles, right axis) of the ion TOF signals. The curves are fits to the Anisimov model [Eq. (6)]. The corresponding values of the aspect ratio $k_{z y}$ are shown close to each fitting curve.

Anisimov interpretation. The average ion energy along the normal to the target surface for different fluencies was also calculated: at low fluence $F_{p} \approx 0.1 \mathrm{~J} / \mathrm{cm}^{2}$ it was $\approx 35 \mathrm{eV}$, and increased to around $100 \mathrm{eV}$ at a maximum fluence $F_{p}$ $\approx 0.8 \mathrm{~J} / \mathrm{cm}^{2}$.

The TOF signals were integrated to find the net ion charge collected by the probe. The angular variation in this quantity is reported in Fig. 8 for both the main Ni plasma and contamination components. As noted above, the distribution of the faster lower- $Z$ contamination signal is wider. A similar effect has been observed in ns laser ablation of $\mathrm{LiNbO}_{3}$ where the $\mathrm{Li}$ had a wider distribution than $\mathrm{Nb}{ }^{32}$ Fitting the angular distributions to Eq. (6), values of $k_{z y} \approx 4.4$ for the aspect ratio of the main $\mathrm{Ni}$ ion feature and $k_{z y} \approx 2.3$ for the contamination component were found. In fact the angular distribution for the integrated overall signal is very close to that of the main peak on its own, since the contamination contribution is $\approx 15 \%$ with respect to the main ion plasma component. Figure 9 shows how $k_{z y}$ and the corresponding angular width $\Delta \theta$ at FWHM [Eq. (8)] of the integrated ion signal vary with laser fluence. The aspect ratio near threshold is $k_{z y} \approx 3.6$ but increases to $k_{z y} \approx 4.7$ at $F_{p} \approx 0.8 \mathrm{~J} / \mathrm{cm}^{2}$. Similar values of the aspect ratio $(\approx 4.4)$ were observed earlier for the ion plasma produced by irradiating bulk $\mathrm{Ni}$ with $\approx 200 \mathrm{fs}, 775 \mathrm{~nm}$ Ti:sapphire laser pulses at a peak fluence of $\approx 4 \mathrm{~J} / \mathrm{cm}^{2} .{ }^{33}$ Instead, larger values of the aspect ratio (from $\approx 6$ to 10 ) were reported when irradiating Ni thin films

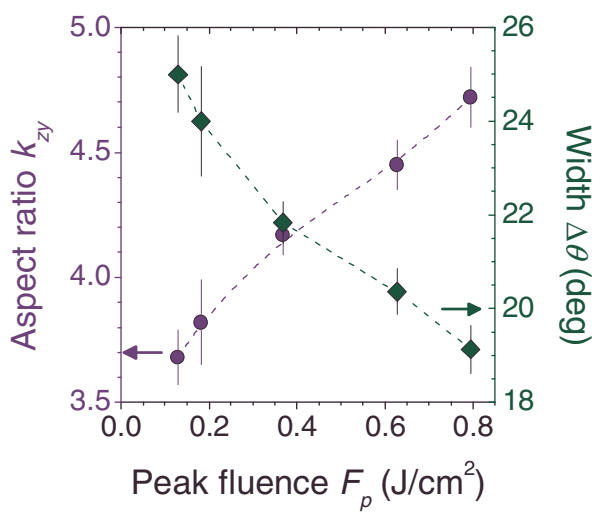

FIG. 9. (Color online) Plasma plume aspect ratio, $k_{z y}$, and corresponding angular width (FWHM) $\Delta \theta$ as a function of peak laser fluence $F_{p}$.

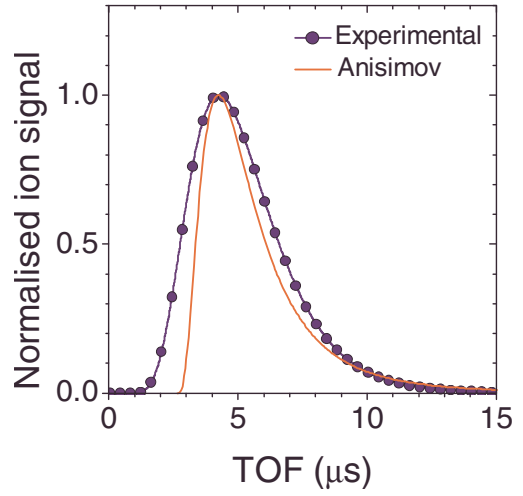

FIG. 10. (Color online) Ion TOF signal (circle) for $F_{p}=0.6 \mathrm{~J} / \mathrm{cm}^{2}$ compared to the Anisimov model prediction for $\gamma=1.25, \varepsilon_{p}=15 \mathrm{eV}$, and $Z_{0}$ $=100 \mathrm{~nm}$ (line).

with thicknesses from 2 to $50 \mathrm{~nm}$ with the same laser pulses at a peak fluence of $1.9 \mathrm{~J} / \mathrm{cm}^{2}$, and an adiabatic index $\gamma$ $\approx 1.27$ was estimated for the $\mathrm{Ni}$ ion plume. ${ }^{19}$ Moreover, a progressive reduction in the aspect ratio was observed as the film thickness increases, thus suggesting that our experimental aspect ratio should correspond to a value $Z_{0}$ of the initial plume larger than $50 \mathrm{~nm}$, which is consistent with the earlier estimate of a 50-200 nm lower limit for $Z_{0}$ based on the propagation of a rarefaction wave through the layer which is vaporized.

By comparing the measured aspect ratios of Fig. 9 with the predictions of the Anisimov model we can get more insights on the thickness $Z_{0}$ of the atomic plasma layer when the adiabatic expansion commences in our experimental conditions. The dependence of $k_{z y}$ on the layer thickness $Z_{0}$ for different values of the adiabatic constant $\gamma$ for a gas cloud with a lateral dimension ratio of $Y_{0} / X_{0}=0.5$ predicted by Anisimov model is shown in Fig. 3 (full symbols). The region corresponding to our measurements lies approximately between $k_{z y}=3.5-4.5$. The $k$-values depend strongly on $\gamma$, the value of which can be rather uncertain. However, for reasonable values of $Z_{0}$, the measured aspect ratios point to a value of $\gamma$ of the order of 1.2-1.3. By taking $\gamma \approx 1.25$ suggests that $Z_{0} \approx 80-400 \mathrm{~nm}$, which are comparable to the earlier estimate based on the propagation of a rarefaction wave through the layer which is vaporized. These values are broadly consistent with MD simulations of ULA ablation of $\mathrm{Ni}$ at $0.6 \mathrm{~J} / \mathrm{cm}^{2}$ by Amoruso et al., ${ }^{5}$ and suggest an order of magnitude of $100 \mathrm{~nm}$ for the typical longitudinal dimension of the atomic plume at the beginning of the 3D expansion described by the Anisimov model.

It is also of interest to consider in detail the shape of the ion TOF signal. The Ni ion signal in the forward direction at $0.6 \mathrm{~J} \mathrm{~cm}^{-2}$ is shown in Fig. 10. The ion energy at the TOF corresponding to the maximum ion flux is $\approx 68 \mathrm{eV}$. This value would correspond to energy per particle $\varepsilon_{p} \approx 15 \mathrm{eV}$ in an Anisimov-type expansion with $\gamma \approx 1.25$. Using these values and $Z_{0} \approx 100 \mathrm{~nm}$ derived from Fig. 3 for $k_{z y} \approx 4.45$ at $F_{p}=0.6 \mathrm{~J} / \mathrm{cm}^{2}$, Eq. (2) yields an ion TOF signal that is somewhat narrower than we observe. This may suggest that the initial plume is rather inhomogeneous with respect to energy per particle. Perhaps it is not surprising that the initial plasma plume is to some extent inhomogeneous for fs pulses 
when we consider that the ion plume properties may reflect the variation in the energy coupled to different parts of the ablation spot and that as the rarefaction wave moves into the heated target, regions lying deeper will unload with lower energy density than regions closer to the surface. These effects are less important in the case of longer ns pulses where a better agreement between experiment and Anisimov predictions was observed. ${ }^{17}$ In this last case, in fact, the interaction of the trailing edge of the laser pulse with the nascent vapor tends to produce a more homogeneous plasma plume as a consequence of plasma absorption.

\section{B. NP plume}

The angular distribution of the overall ablation plume was recorded by making deposits on curved transparent substrates located at $7.7 \mathrm{~cm}$ from the target and measuring the spatial distribution of thickness by optical transmission. The number of laser shots was varied according to the fluence used. Since the NP plume constitutes the major part of the ablated material at the moderate laser fluencies used in the present investigation, the deposition profile is close to that of the NPs and can be considered as representative of the NP plume angular distribution. The physical description of the expansion of the NP material produced in ULA to dimensions much larger than the ablation depth or spot size has not been investigated in detail. In the following, we aim to explore if an Anismov-type description is appropriate also for this important component of the ULA process and what value of the adiabatic index or initial layer thickness of the expanding NP plume should be used.

As an example, Figs. 11(a) and 11(b) show twodimensional (2D) maps of the film thickness per pulse, $t$, at (a) low $\left(F_{p}=0.15 \mathrm{~J} / \mathrm{cm}^{2}\right)$ and (b) high fluence $\left(F_{p}\right.$ $\left.=0.7 \mathrm{~J} / \mathrm{cm}^{2}\right)$. For the deposition at $F_{p}=0.15 \mathrm{~J} / \mathrm{cm}^{2} 118800$ shots were used, while at $F_{p}=0.7 \mathrm{~J} / \mathrm{cm}^{2} 15840$ shots were fired at the Ni target. We observe a broadening of the deposit thickness as the fluence increases. Figure 12(a) shows the angular variation in the film thickness in the $z y$ plane, at $F_{p}=0.15 \mathrm{~J} / \mathrm{cm}^{2}$. A fit using the Anisimov distribution [Eq. (6)] is also reported in Fig. 12(a), and describes very well the experimental data, with a $k_{z y}$ value of 9.5. This can be compared to the value of $\approx 3.7$ observed for the ion plasma plume at the same fluence, which indicates a more forwardpeaked expansion of the NP component with respect to the ion plume. A rather good agreement between the Anisimov model and the experimental angular distribution is maintained up to fluencies of $\approx 0.3-0.4 \mathrm{~J} / \mathrm{cm}^{2}$. A similar behavior was observed also for the angular profiles in the $z x$ plane.

The angular distribution at larger fluencies is distinctly different and is not very well-described by the Anisimov distribution. As an example, Fig. 12(b) reports the angular variation in the film thickness at $F_{p}=0.7 \mathrm{~J} / \mathrm{cm}^{2}$. We can observe quite similar angular widths at FWHM for model and experimental data, but the measured distribution is somewhat broader than expected at small angles, while becoming narrower on the wings at larger angles.

The deposits on the hemispherical substrate (see Fig. 1) allow us to measure the angular profiles in both the $z x$ and $z y$
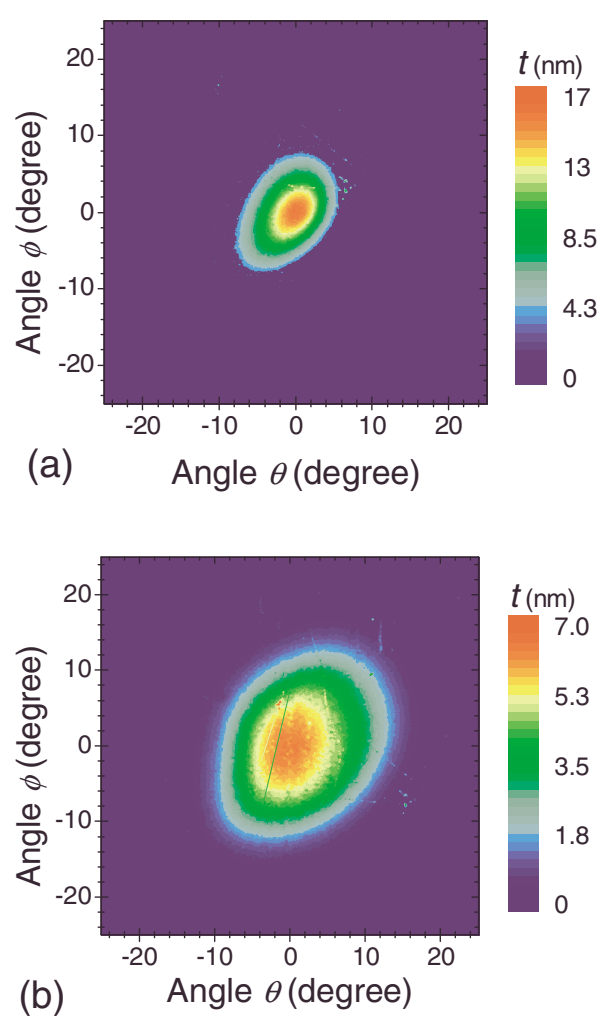

FIG. 11. (Color online) 2D maps of the films thickness per pulse $t$ at (a) low $\left(F_{p}=0.15 \mathrm{~J} / \mathrm{cm}^{2}, 118800\right.$ shots $)$ and (b) high-fluence $\left(F_{p}=0.7 \mathrm{~J} / \mathrm{cm}^{2}\right.$ 15840 shots).

planes. In Fig. 13 we report the FWHM of the angular distributions measured in the $z y(\Delta \theta)$ and $z x(\Delta \phi)$ planes as a function of laser peak fluence $F_{p}$. We observe slightly wider distributions in the $z y$ plane reflecting the fact that the minor radius of the elliptical ablation spot lies along the $y$ axis. This observation indicates that the flip-over effect predicted by the Anisimov model occurs also for the expansion of a NP

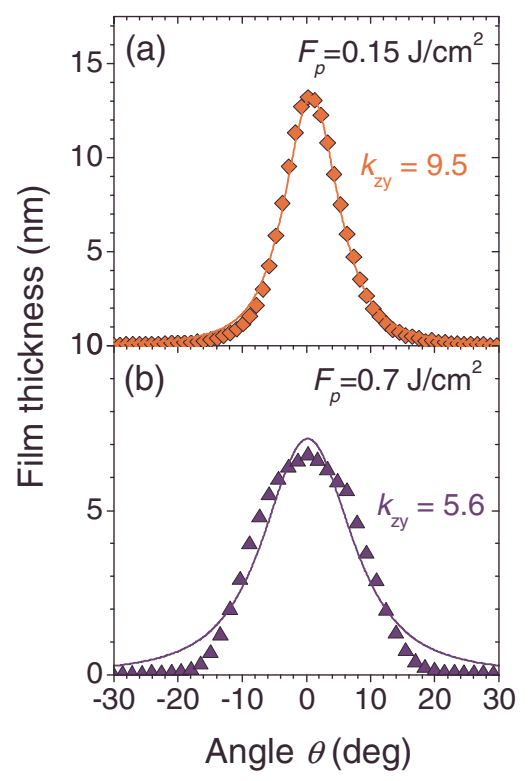

FIG. 12. (Color online) Angular distribution of deposited material for a peak fluence (a) $F_{p}=0.15 \mathrm{~J} / \mathrm{cm}^{2}$ and (b) $F_{p}=0.7 \mathrm{~J} / \mathrm{cm}^{2}$. The solid lines are best fits to the respective data sets using Eq. (6); the corresponding $k_{z y}$ value is indicated in the figure. 


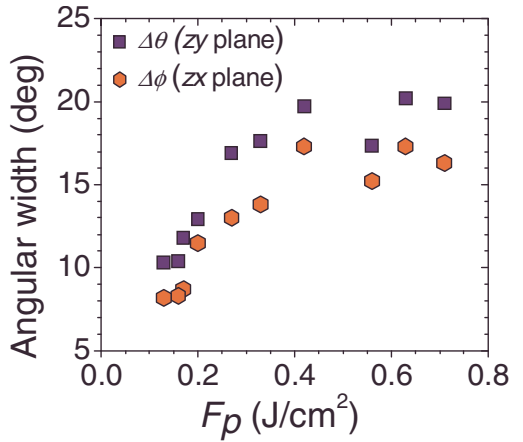

FIG. 13. (Color online) Angular widths $\Delta \theta$ ( $z y$ plane) and $\Delta \phi$ ( $z x$ plane) of the deposits as a function of the peak fluence $F_{p}$.

plume. This flip-over effect consists in the fact that when the plume is produced in vacuum by an elliptical beam spot, in the inertial stage of expansion its major axis lies at $90^{\circ}$ to that of the initial spot. ${ }^{16}$ This is explained on the basis of initial pressure gradients.

The width of the angular distribution as a function of the peak fluence $F_{p}$ in Fig. 13 shows an increase in the region 0.1 to $0.4 \mathrm{~J} / \mathrm{cm}^{2}$ and remains rather constant above $0.4 \mathrm{~J} / \mathrm{cm}^{2}$. In comparison to the behavior of the ion plasma angular width reported in Fig. 9, we observe a rather different influence of the laser fluence on the NPs. The NP plume initially tends to broaden as $F_{p}$ increases, then levelling off at laser fluencies above $F_{p} \approx 0.4 \mathrm{~J} / \mathrm{cm}^{2}$. Instead, the width of the ion plume angular distribution reduces monotonically with $F_{p}$. This suggests that the observed changes in the NP plume distribution at larger fluencies is not a feature of the whole ablated material, rather it is mostly related to a mechanism which seems to influence only the NP dynamics. A complete understanding of such a mechanism is not yet clear at this stage and deserves further experimental investigation and theoretical analysis of the first stages of NP material expansion by hydrodynamic or MD modeling. However, even at this stage, it is possible to provide some comments on possible explanations, as described below.

Changes in the deposit profile with increasing laser pulse energy could be due to self-sputtering produced by energetic species impinging the substrate. In fact, we observe an increase in the ion average kinetic energy with $F_{p}$, and the ion energies measured by the ion probe are sufficient to cause self-sputtering of the growing film. This effect will be most pronounced in the center of the profile where the ion energies are highest; which would act to flatten the profile, as is indeed observed in Fig. 12(b). However, we also observe a reduced deposition rate at larger angles with respect to Anisimov prediction. Since both energy and amount of impinging ions is progressively reduced at larger angle $\theta$, as shown in Figs. 7(b) and 8, the self-sputtering efficiency should be less at these angles, contrary to the experimental observation. Moreover, it seems that the ion fraction is too small to cause a significant effect on the final deposition profile, thus, we can rule out this mechanism.

The previous comment, therefore, suggests that the cause of the changes should be related to a variation in the NP expansion dynamics before deposition. In this respect, in recent experimental investigation and hydrodynamic model- ing of ULA with two temporally delayed laser pulses, it was observed that absorption of the second pulse in the nascent atomic plume induced by the first pulse produces a plasma which generates a pressure pulse that acts to impede the outflow of underlying target material, which subsequently decomposes for the most part in form of NPs, finally reducing the ablation depth. ${ }^{20,34}$ Therefore, we might consider if the pressure exerted by the nascent atomic plasma plume can somehow affect the expansion dynamics of the deeper layer of irradiated material which finally produces the NP plume in the initial stages of the ablation process.

The average pressure, $\langle p\rangle$, of the ULA atomic plasma at the early stage of its formation and expansion can be estimated as ${ }^{15,28}$

$$
\langle p\rangle \approx(\gamma-1) \varepsilon_{p} n_{p},
$$

where $n_{p}, \varepsilon_{p}$, and $\gamma$ are the number density, the average energy per particle, and the adiabatic index of the atomic plasma. The adiabatic index $\gamma$ of the partly ionized atomic plasmas was shown to vary in the range 1.2-1.3. The atomic plume accounts for only $10 \%-20 \%$ of the total ablated mass, ${ }^{3,5,12,13}$ which corresponds to a surface layer decomposing into atomic form with a typical thickness $d_{a t}$ of several nanometers, for maximum ablation depth of the craters of several tens of nanometers observed in our experiment. This leads to an average number density of the atomic plasma $n_{p}$ at the electron-phonon relaxation time $\tau_{e p}$

$$
n_{p} \approx n_{s}\left(d_{a t} / u \tau_{e p}\right) \text {, }
$$

where $n_{s}$ and $u$ are solid atomic number density of nickel $\left(9.1 \times 10^{22} \mathrm{~cm}^{-3}\right)$ and atomic expansion velocity, respectively. The electron-phonon relaxation time of $\mathrm{Ni}$ is $\tau_{e p}$ $\approx 6-7$ ps. ${ }^{9,35}$ By energy balance considerations, the average energy per particle $\varepsilon_{p}$ can be estimated as

$$
\varepsilon_{p} \approx \frac{(1-R) \alpha F}{n_{s}},
$$

where $R$ is the target reflectivity $\left(0.51\right.$ at $45^{\circ}$ incidence for $p$-polarized light) and $\alpha$ is the linear absorption coefficient of nickel $\left(7.4 \times 10^{5} \mathrm{~cm}^{-1}\right)$. In our experimental conditions, $\varepsilon_{p}$ is expected to vary from few electronvolts to $\approx 10 \mathrm{eV}$ as a function of the laser fluence, with corresponding velocity $u$ $=\left(2 \varepsilon_{p} / \mathrm{m}\right)^{1 / 2}$ ranging from $2 \times 10^{5}$ to $6 \times 10^{5} \mathrm{~cm} / \mathrm{s}$. These values are consistent with the observed average ion velocity and with earlier theoretical model predictions and experimental results. ${ }^{5,6}$ Considering an initial thickness of the surface layer decomposing into the atomic plume $d_{a t}$ ranging from $\approx 2$ to $5 \mathrm{~nm}$ and an adiabatic index of the atomic plume $\gamma \approx 1.25$, we obtain average pressure $\langle p\rangle$ of the atomic component of the order of $0.5-1.5 \mathrm{GPa}$ at $F \approx 0.1 \mathrm{~J} / \mathrm{cm}^{2}$, rising up with the fluence to $\approx 2-6 \mathrm{GPa}$ at $F \approx 0.8 \mathrm{~J} / \mathrm{cm}^{2}$. These values should be compared with the pressure in the underlying material decomposing into NPs at this time. NPs are produced by mechanical fragmentation and phase explosion occurring around the critical point, the critical pressure of $\mathrm{Ni}$ $(\approx 1.1 \mathrm{GPa})$ can be considered as indicative of the pressure of this relaxing material. Therefore, we can expect that the atomic plasma pressure might have a progressively larger influence on the hydrodynamic evolution of the NP material 
as the fluence increases, which could explain the changes in the NP plume expansion dynamics we observe at $F_{p}$ larger than about $0.3 \mathrm{~J} / \mathrm{cm}^{2}$, though detailed hydrodynamic modeling will be necessary to fully confirm this interpretation. Very recently Zhigilei et al. ${ }^{36}$ have confirmed by MD simulation that low velocity NPs emitted during the ablation process can be pushed back and redeposited on the target due to the plasma pressure. This correlates well with our interpretation of the change in the NP plume expansion with increasing fluence.

Finally, as we observe angular distributions in fairly good agreement with the Anisimov model at low fluence, we can deduce some information on adiabatic index and initial layer thickness of the expanding NP plume for this case. At $F_{p} \approx 0.1-0.3 \mathrm{~J} / \mathrm{cm}^{2}$, the measured maximum ablation depth varies in the range $20-60 \mathrm{~nm}$. MD simulations of ULA indicate that when the material at the limit of ablation undergoes phase decomposition, the overall density of the NP material is a few times lower than solid density, thus an initial thickness of the NP layer in an Anisimov-type expansion roughly equal to a few times the ablation depth can be inferred, i.e., in the range $50-200 \mathrm{~nm}$. This corresponds to a $Z_{0} / X_{0}$ lying in the interval $(2.5-10) \times 10^{-4}$. For $F_{p} \approx 0.1-0.3 \mathrm{~J} / \mathrm{cm}^{2}$, we observe that $k_{z y}$ reduces from $\approx 10$ to 7 as a function of the fluence, which suggests a value of the adiabatic index $\gamma$ $\approx 1.4$ for the NP plume produced in the ULA process at moderate fluence. However, we are not aware of any other experimental or theoretical estimate for the appropriate $\gamma$-value to describe the gas dynamics of NP material and this value should be considered as indicative.

\section{ABLATION THRESHOLD AND ABLATION RATE}

An important ULA issue is to understand how ablation threshold and ablation rate versus fluence are related to the material and laser pulse parameters. For metals, the TTM is widely employed to describe temporal and spatial evolution of electron and lattice temperatures. ${ }^{1}$ Simpler theoretical approaches to laser ablation couple TTM to assumptions about the kinetics of matter emission, ${ }^{21,22,37}$ while MD and HM methods combined with TTM are employed for more quantitative descriptions of the process. ${ }^{3-9}$ These studies are often complicated by the fact that the physical parameters involved (electron-phonon coupling coefficient and electron thermal conductivity, e.g.) in the highly nonequilibrium regime induced by ultrashort laser irradiation are in general not very well known and still under investigation, ${ }^{38}$ and approximation should be normally used. Also, the analysis of material relaxation and removal after irradiation have shown the simultaneous presence of different mechanisms including vaporization, fragmentation, and phase explosion, ${ }^{3-9}$ which finally give rise to a complex plume, as discussed in previous sections. All these issues continue to stimulate analysis of ultrafast laser ablation even in the relatively simple case of metallic targets. In the following we discuss our results on the ablation threshold of $\mathrm{Ni}$ and on the variation in the maximum ablation depth as a function of the laser fluence.
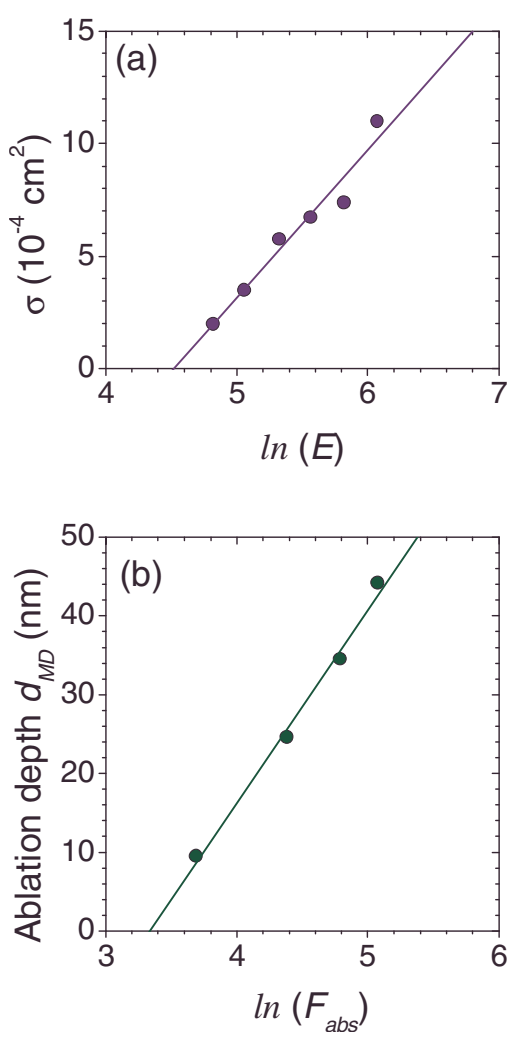

FIG. 14. (Color online) (a) Variation in the beam spot size $\sigma$ on the logarithmic of the pulse energy $\ln (E)$, (with the energy $E$ measured in microjoule). The slope of the linear fit is used to find the energy threshold $E_{t h}$ and the focused Gaussian beam size $\sigma_{0}$. (b) Variation in the ablation depth $d_{\mathrm{MD}}$ as a function of the logarithmic of the absorbed laser pulse fluence $\ln \left(F_{a b s}\right)$ (with $F_{a b s}$ in $\mu \mathrm{J} / \mathrm{cm}^{2}$ ) predicted by MD simulations of Ni irradiated by 527 $\mathrm{nm}, \approx 300 \mathrm{fs}$ laser pulses in Ref. 5 .

\section{A. Ablation threshold}

The ablation threshold of $\mathrm{Ni}$ has been estimated through the measurements of the beam spot size $\sigma$ at various laser pulse energies. ${ }^{23}$ For a Gaussian beam, the area over which the pulse energy exceeds the threshold energy, is given by

$$
\sigma=\frac{\sigma_{0}}{2} \ln \left(\frac{E}{E_{t h}}\right),
$$

where $\sigma_{0}$ is the area of the Gaussian beam at the $1 / \mathrm{e}^{2}$ point on the energy distribution, $E$ is the laser pulse energy, and $E_{t h}$ is the threshold energy to damage/ablate the target. This dependence has been also seen in recent MD simulations of the crater formation during ablation induced by a laser pulse with a Gaussian spatial distribution of energy. ${ }^{39}$ Two laser shots were fired at each location. Figure 14(a) shows the variation in $\sigma$ as a function of $\ln (E)$ (with $E$ measured in microjoule), from which a threshold energy $E_{t h} \approx 80 \mu \mathrm{J}$ and a beam spot $\sigma_{0}=1.3 \times 10^{-3} \mathrm{~cm}^{2}$ are obtained. The corresponding average threshold fluence is $F_{t h}=E_{t h} / \sigma_{0}$ $\approx 60 \mathrm{~mJ} / \mathrm{cm}^{2}$.

Figure 14(b) reports the variation in the ablation depth $d_{\mathrm{MD}}$ as a function of the logarithmic of the absorbed laser pulse fluence $F_{a b s}$ predicted by MD simulations of Ni irradiated by $527 \mathrm{~nm}, \approx 300$ fs laser pulses obtained earlier by Amoruso et $a .^{5}$ By extrapolation to $d_{\mathrm{MD}}=0$, an absorbed threshold fluence of $F_{a b s, t h}=28 \mathrm{~mJ} / \mathrm{cm}^{2}$ is obtained, which, 
for $45^{\circ}$ incidence $p$-polarized light $(R=0.51)$, corresponds to $F_{t h, \mathrm{MD}}=F_{a b s} /(1-\mathrm{R}) \approx 57 \mathrm{~mJ} / \mathrm{cm}^{2}$, in fairly good agreement with that obtained by the slope method applied to the spot size in Fig. 14(a). This is also consistent with earlier estimates based on the plume emission analysis and crater depth measurements for normal incidence. ${ }^{5}$ It is also interesting to note that MD simulations of Fig. 14(b) give a fluence logarithmic dependence of the ablation depth $d_{\mathrm{MD}}$ $=d_{0} \ln \left(F_{a b s} / F_{a b s, t h}\right)$, with a constant $d_{0}=(25 \pm 2)$. Studying melting threshold of Ni films, Wellershoff et al. ${ }^{36}$ measured a diffusive penetration length of excited electrons for $\mathrm{Ni}$ during the time of electron-phonon equilibration $L_{c}=31 \mathrm{~nm}$. The fact that this value is similar to $d_{0}$ seems to suggest that for $\mathrm{Ni}$, in our experimental conditions, the electron diffusion is always dominant even for fluence values around the ablation threshold.

For the sake of completeness, we would like to observe that with the assumption that vaporization is the material removal mechanism, the ablation threshold $F_{t h}$ was estimated earlier as

$$
F_{t h}=\frac{\rho \Omega_{\mathrm{vap}} l_{0}}{(1-R)},
$$

where $\rho$ is the material solid density, $\Omega_{\text {vap }}$ is the specific (per unit mass) heat of evaporation, and $l_{0}$ is a characteristic length over which energy is spread in the material. ${ }^{21}$ Two regimes were considered: (i) a low-fluence regime for the case when the number density of electrons is so low that energy transfer occurs only within the area characterized by the optical skin depth $\delta=1 / \alpha$ ( $\alpha$ is the optical absorption coefficient of the material) and (ii) a high-fluence regime occurring when electron thermal diffusion dominates. In the former case $l_{0}=\delta$, while in the latter case $l_{0}=l_{e, t h}$, the electron thermal diffusion length. The optical absorption depth of $\mathrm{Ni}$ at $527 \mathrm{~nm}$ is $\delta=13.5 \mathrm{~nm}$ and Eq. (13) predicts an average ablation threshold fluence for the low-fluence regime of $\approx 150 \mathrm{~mJ} / \mathrm{cm}^{2}$, which is larger than our experimental estimate of $\approx 60 \mathrm{~mJ} / \mathrm{cm}^{2}$. Moreover, the use of $l_{0} \approx d_{0}$ or $L_{c}$ in Eq. (13) would lead to an even larger estimate of the fluence threshold, contrary to what is experimentally observed. This indicates that assumption of complete vaporization of a layer of thickness $l_{0}$ overestimates the fluence threshold. Since in fs laser ablation part of the material is released in aggregated form, a lower threshold fluence should be expected, as indeed is observed experimentally.

\section{B. Maximum ablation depth versus fluence}

Logarithmic dependence of the ablation depth $d$ on the laser fluence have been predicted earlier by using the TTM approach $^{21}$

$$
d=l_{0} \ln \left(\frac{F}{F_{t h}}\right) .
$$

This logarithmic dependence is also predicted, in the fluence range of concern to the present experiment, by the results of MD simulations for Ni ablation of Ref. 5, and reported in Fig. 14(b). More recently an approximately logarithmic dependence of the ablation depth on the laser fluence has been

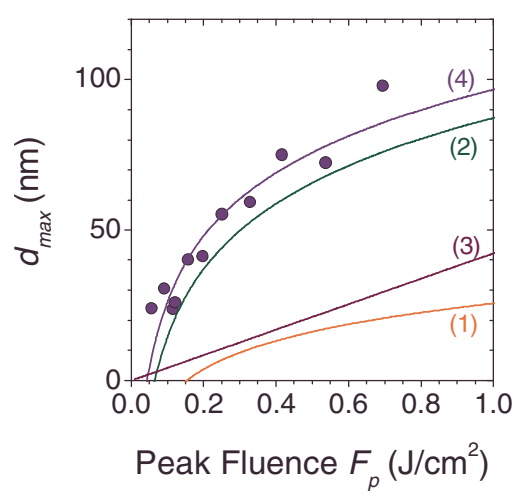

FIG. 15. (Color online) Maximum ablation depth, $d_{\max }$, as a function of the peak fluence $F_{p}$ (circles). Curves: (1) maximum ablation depth calculated according to Eq. (14) in the low-fluence regine $\left[l_{0}=\delta, F_{\text {th }}\right.$ given by Eq. (13)]; (2) maximum ablation depth calculated according to Eq. (15) in the diffusive regime, with $l_{0}=L_{c}$ and $F_{t h}=60 \mathrm{~mJ} / \mathrm{cm}^{2}$; (3) linear dependence predicted by Vestentoft and Balling model [Eq. (16)]; and (4) fit of the experimental data to Eq. (15) with $l_{0}$ and $F_{t h}$ as free parameters $\left(l_{0}\right.$ $\left.=(30 \pm 3) \mathrm{nm}, F_{t h} \approx 40 \mathrm{~mJ} / \mathrm{cm}^{2}\right)$.

also reported in studies of craters formed during ULA in the thermal regime based on MD simulations. ${ }^{39}$

Figure 15 reports the maximum ablation depth, $d_{\max }$, obtained by Zygo analysis of the ablation craters as a function of the peak fluence $F_{p}$. In the following, we consider that $d_{\text {max }}$ represents the ablation depth corresponding to the maximum value of the fluence within the laser beam spot, therefore the following relationship is expected:

$$
d_{\max }=l_{0} \ln \left(\frac{F_{p}}{F_{t h}}\right) .
$$

For comparison, in Fig. 15 we report the calculated ablation depth according to Eq. (14) in the low-fluence regime (curve 1 ), with $F_{t h}$ estimated from Eq. (13). ${ }^{21}$ The assumption of energy absorption only within the optical skin depth leads to estimates of the maximum ablation depth which falls well below the experimental values, again confirming that in our case we are in a diffusive regime. If we consider the diffusive regime with $l_{0}=L_{c}$ and the experimental fluence threshold obtained above with the slope method $F_{t h}=60 \mathrm{~mJ} / \mathrm{cm}^{2}$ (curve 2), we get estimates which are much closer to the experimental values, even if $d_{\max }$ remains still slightly underestimated.

An alternative model based on the electron conduction in the high-fluence regime was considered by Vestentoft and Balling $^{22}$ observing that the ablation time depends on the fluence and obtaining a different, linear relationship between maximum ablation depth and fluence which in our case reads

$$
d_{\max }=\sqrt{\frac{2}{\pi e}} \frac{(1-R) F_{p}}{\rho \Omega_{\mathrm{vap}}},
$$

where $e$ is the Neper number. This is also shown for completeness in Fig. 15 (curve 3), and its estimates also fall well below the experimental measurements.

Our previous analysis points to the fact that the variation in the maximum ablation depth seems to be better described by a logarithmic dependence on the laser pulse fluence, which can be understood if one considers that the tempera- 
ture profiles approximately follow an exponential dependence as a function of the depth inside the material. ${ }^{5-7}$ For this reason, in Fig. 15 we have finally fitted the experimental data to the logarithmic dependence of Eq. (15) with $F_{t h}$ and $l_{0}$ as free parameters. The fit is shown in Fig. 15 as curve 4 and it describes fairly well the experimental measurements with $F_{t h} \approx 40 \mathrm{~mJ} / \mathrm{cm}^{2}$ and $l_{0}=(30 \pm 3) \mathrm{nm}$. The value of $l_{0}$ obtained from the fitting procedure is consistent with the diffusive electron length $L_{c}$ of $\mathrm{Ni}$, while the fluence threshold is about $30 \%$ less than that obtained by the slope method in Sec. V A. This can be understood if we consider that the data of Fig. 15 refers to multishot ablation experiments which can be influenced by incubation effects and changes in the target reflectivity due to the progressive variation in the target surface optical properties during irradiation. Taking into account the incubation coefficient of $\mathrm{Ni}(\mathrm{S}=0.965),{ }^{40}$ a reduction in about $20 \%$ is expected for the ablation threshold fluence for the multishot irradiation with several hundreds of pulses used in our experiments.

In conclusion, our analysis seems to indicate that a logarithmic dependence is well-suited for the description of the variation in the ablation depth on the laser fluence, in the case of $\mathrm{Ni}$ in a fluence regime from ablation threshold up to $\approx 1 \mathrm{~J} / \mathrm{cm}^{2}$. This is related to the conduction of the absorbed energy by the heated electrons to distances of the order of the diffusive electron depth $L_{c}$ on the timescale of electronphonon equilibration, as also indicated by earlier MD results. ${ }^{5}$ The fluence threshold for ablation is, indeed, reduced with respect to that predicted in early model of ULA based on the occurrence of complete vaporization of the irradiated material. This is related to the fact that relaxation of the excited material follows more complex thermodynamic paths, and material removal mostly occurs through fragmentation and phase explosion, which leads to the significant presence of nanoparticulates observed in ULA deposition experiments.

\section{CONCLUSIONS}

In this investigation, experiments aiming to examine various aspects of the ULA of a metallic target $(\mathrm{Ni})$ in high vacuum were carried out with the aim of addressing both expansion dynamics of the various ULA plume components and basic properties of the ablation process. The ionic plasma plume was analyzed using a Langmuir ion probe, obtaining both ion flux temporal profiles and ion angular distribution. Since the atomic component constitutes a minor fraction of the ablated material, the angular distribution of the nanoparticulate component produced during ULA was characterized by measuring the thickness map of deposition on a transparent substrate. Moreover, the ablated material per pulse was found by applying scanning white light interferometry to measure the craters produced on a stationary target.

Our results indicate that the angular distribution of both the ionic and NP components can be generally described by the Anisimov model. The Anisimov model describes the ion angular distribution at any laser fluence, in the investigated range from ablation threshold to $\approx 1 \mathrm{~J} / \mathrm{cm}^{2}$. Instead, the NP plume angular distribution is well-described by the Anisimov model only at fluencies lower than $\approx 0.4 \mathrm{~J} / \mathrm{cm}^{2}$. At larger fluencies, we observe quite similar angular widths at FWHM for model and experimental data, but the experimental distribution is somewhat broader than expected at small angles, and narrower on the wings at larger angles. This effect was interpreted in terms of the influence of the pressure exerted by the nascent atomic plasma plume on the initial hydrodynamic evolution of the NPs. Moreover, we find that the flipover effect which was earlier observed for ablation plumes produced with ns laser pulses, also occurs in the case of the NPs produced during the ULA process.

Analysis of the measured plume aspect ratio in the frame of the Anisimov model suggests that the 3D expansion of the ion plume, considered here as representative of the atomic ablation component, starts on a timescale comparable to the time needed for the propagation of a rarefaction wave through the layer which is vaporized. Moreover, an adiabatic index of the ion plasma of the order of 1.2-1.3 has been inferred. Similar comparison for the NP plume at fluencies of $0.1-0.3 \mathrm{~J} / \mathrm{cm}^{2}$ suggests a value of the adiabatic index $\gamma$ $\approx 1.4$ for this component. However, we are not aware of any other experimental or theoretical estimates for the appropriate $\gamma$-value to describe the gas dynamics of NP material, and this value should be considered as indicative.

Comparison between total ablated mass, obtained by crater depth maps measurements, and deposited material yield showed a fairly good agreement, further confirming the observation that the ablated material in ULA predominately decomposes as NPs, while the atomic products constitute a minor component.

Analysis of the fluence threshold and maximum ablation depth was also carried out and compared to predictions of theoretical models. Our results indicate that in the case of $\mathrm{Ni}$ the absorbed energy is spread over a range comparable with the diffusive electron depth $L_{c}$ on the timescale of electronphonon equilibration and a logarithmic dependence is wellsuited for the description of the variation in the ablation depth on the laser fluence, from ablation threshold up to $\approx 1 \mathrm{~J} / \mathrm{cm}^{2}$. The fluence threshold for ablation is, indeed, reduced with respect to that predicted in phenomenological TTM approaches of ULA based on complete vaporization of the irradiated material, as a consequence of the more complex mechanisms (fragmentation and phase explosion, e.g.) involved in the material removal.

We consider that several of the features observed in the case of $\mathrm{Ni}$ can be reasonably considered as general characteristics of the ablation process and of the related expansion dynamics for any metal irradiated by ultrashort laser pulses at moderate intensities.

\section{ACKNOWLEDGMENTS}

This collaborative experiment was partly funded by the Istituto Italiano di Cultura, Dublin, the Royal Irish Academy and the Human Resources Development Foundation of Tianjin. We also acknowledge support from Science Foundation Ireland under Contract No. 07/IN.1/I1771.

${ }^{1}$ S. I. Anisimov, B. L. Kapelovich, and T. L. Perel'man, Zh. Eksp. Teor. 
Fiz. 66, 776 (1974).

${ }^{2}$ A. M. Komashko, M. D. Feit, and A. M. Rubenchik, Proc. SPIE 3935, 97 (2000).

${ }^{3}$ M. E. Povarnitsyn, T. E. Itina, M. Sentis, K. V. Khishchenko, and P. R. Levashov, Phys. Rev. B 75, 235414 (2007).

${ }^{4}$ J. P. Colombier, P. Combis, R. Stoian, and E. Audouard, Phys. Rev. B 75, 104105 (2007).

${ }^{5}$ S. Amoruso, R. Bruzzese, X. Wang, N. N. Nedialkov, and P. A. Atanasov, J. Phys. D: Appl. Phys. 40, 331 (2007).

${ }^{6}$ C. Cheng and X. Xu, Phys. Rev. B 72, 165415 (2005).

${ }^{7}$ D. Perez and L. J. Lewis, Phys. Rev. B 67, 184102 (2003).

${ }^{8}$ P. Lorazo, L. J. Lewis, and M. Meunier, Phys. Rev. Lett. 91, 225502 (2003).

${ }^{9}$ D. S. Ivanov and L. V. Zhigilei, Phys. Rev. B 68, 064114 (2003).

${ }^{10}$ S. Amoruso, R. Bruzzese, N. Spinelli, R. Velotta, M. Vitiello, X. Wang, G. Ausanio, V. Iannotti, and L. Lanotte, Appl. Phys. Lett. 84, 4502 (2004).

${ }^{11}$ S. Amoruso, R. Bruzzese, C. Pagano, and X. Wang, Appl. Phys. A: Mater. Sci. Process. 89, 1017 (2007).

${ }^{12}$ S. Amoruso, R. Bruzzese, X. Wang, and J. Xia, Appl. Phys. Lett. 92, 041503 (2008).

${ }^{13}$ S. Noël and J. Hermann, Appl. Phys. Lett. 94, 053120 (2009).

${ }^{14}$ K. Oguri, Y. Okano, T. Nishikawa, and H. Nakano, Phys. Rev. B 79, 144106 (2009).

${ }^{15}$ S. I. Anisimov, D. Bauerle, and B. S. Luk'yanchuk, Phys. Rev. B 48, 12076 (1993).

${ }^{16}$ S. I. Anisimov, B. S. Luk'yanchuk, and A. Luches, Appl. Surf. Sci. 96-98, 24 (1996)

${ }^{17}$ T. N. Hansen, J. Schou, and J. G. Lunney, Appl. Phys. A: Mater. Sci. Process. 69, S601 (1999).

${ }^{18}$ B. Toftmann, J. Schou, and J. G. Lunney, Phys. Rev. B 67, 104101 (2003).

${ }^{19}$ G. O. Williams, S. Favre, and G. M. O'Connor, Appl. Phys. Lett. 94, 101503 (2009).

${ }^{20}$ T. Donnelly, J. G. Lunney, S. Amoruso, R. Brusezze, X. Wang, and X. Ni, J. Appl. Phys. 106, 013304 (2009).
${ }^{21}$ S. Nolte, C. Momma, H. Jacobs, A. Tunnermann, B. N. Chichkov, B. Wellegehausen, and H. Welling, J. Opt. Soc. Am. B 14, 2716 (1997).

${ }^{22}$ K. Vestentoft and P. Balling, Appl. Phys. A: Mater. Sci. Process. 84, 207 (2006).

${ }^{23}$ J. M. Liu, Opt. Lett. 7, 196 (1982).

${ }^{24}$ B. Doggett and J. G. Lunney, J. Appl. Phys. 105, 033306 (2009).

${ }^{25}$ R. K. Singh and J. Narayan, Phys. Rev. B 41, 8843 (1990).

${ }^{26}$ J. G. Lunney, B. Doggett, and Y. Kaufman, J. Phys.: Conf. Ser. 59, 470 (2007).

${ }^{27}$ Y. B. Zel'dovich and Y. P. Raizer, Physics of Shock Waves and High Temperature Hydrodynamic Phenomena (Dover, New York, 2002).

${ }^{28}$ G. O. Williams, G. M. O'Connor, P. T. Mannion, and T. J. Glynn, Appl. Surf. Sci. 254, 5921 (2008).

${ }^{29}$ B. Toftmann, B. Doggett, J. Schou, and J. G. Lunney, (unpublished).

${ }^{30}$ X. Wang, S. Amoruso, and J. Xia, Appl. Surf. Sci. 255, 5211 (2009).

${ }^{31}$ T. Donnelly, B. Doggett, and J. G. Lunney, Appl. Surf. Sci. 252, 4445 (2006).

${ }^{32}$ S. Canulescu, E. L. Papadopoulou, D. Anglos, T. Lippert, C. W. Schneider, and A. Wokaun, J. Appl. Phys. 105, 063107 (2009).

${ }^{33}$ P. T. Mannion, S. Favre, C. Mullan, D. S. Ivanov, G. M. O'Connor, T. J. Glynn, B. Doggett, and J. G. Lunney, J. Phys.: Conf. Ser. 59, 753 (2007).

${ }^{34}$ M. E. Povarnitsyn, T. E. Itina, K. V. Khishchenko, and P. R. Levashov, Phys. Rev. Lett. 103, 195002 (2009).

${ }^{35}$ S.-S. Wellershoff, J. Hohlfeld, J. Güdde, and E. Matthias, Appl. Phys. A: Mater. Sci. Process. 69, S99 (1999).

${ }^{36}$ L. V. Zhigilei, Z. Lin, and D. S. Ivanov, J. Phys. Chem. C 113, 11892 (2009).

${ }^{37}$ K. Furusawa, K. Takahashi, H. Kumagai, K. Midorikawa, and M. Obara, Appl. Phys. A: Mater. Sci. Process. 69, S359 (1999).

${ }^{38}$ Z. Lin, L. Zhigilei, and V. Celli, Phys. Rev. B 77, 075133 (2008).

${ }^{39}$ D. Bouilly, D. Perez, and L. J. Lewis, Phys. Rev. B 76, 184119 (2007).

${ }^{40}$ J. Güdde, J. Hohlfeld, J. G. Müller, and E. Matthias, Appl. Surf. Sci. 127-129, 40 (1998). 